\title{
Autoabandono del tabaco en una muestra española. Un estudio exploratorio
}

\section{Self-quitting in a Spanish sample. An exploratory study}

\author{
Bartolomé Marín Romero*; Jesús Gil Roales-Nieto**; Emilio Moreno San Pedro*. \\ * Universidad de Huelva; ** Universidad de Almería.
}

$\mathrm{E}$ 1 consumo de tabaco es un grave problema de salud pública, debido fundamentalmente a su relación con enfermedades cardiovasculares y con distintos tipos de cáncer (Bjartveit y Tverdal, 2009). Con el objetivo de reducir la prevalencia del tabaquismo, se han probado diferentes tipos de tratamiento, tanto farmacológicos como psicológicos o una combinación de ambos, no obstante, la cifra de éxito de dichos tratamientos es muy variable según distintos estudios (Raich et al., 2015; Thomsen, Villebro, y Moller, 2014). Menos estudiado ha sido el abandono del consumo de tabaco sin ayuda profesional (autoabandono o self-quitting, en terminología inglesa), siendo este el principal método de cesación tabáquica usado por la mayoría de los fumadores que intentan abandonar el consumo de tabaco, llegando a estimar que el $95 \%$ de los fumadores tienen éxito con este método (Schater, 1990). Otros estudiosos del tema aportan cifras más modestas que van desde el $54 \%$ hasta el $69 \%$ de fumadores que han conseguido el cese del uso de tabaco mediante autoabandono (Smith, Chapman, y Dunlop, 2015). Se pretende realizar con el presente estudio, un análisis exploratorio de variables que puedan ayudar a explicar el éxito o fracaso en cesación tabáquica mediante autoabandono. El presente estudio obtuvo el informe favorable de la Comisión de Bioética de la Universidad de Almería, cuyo número de referencia es UALBIO2011/025.

Los participantes del estudio se consiguieron mediante anuncios en prensa y radio y centros de salud, se les realizó una entrevista dirigida al registro de distintas variables relacionadas con el consumo de tabaco, edad de inicio, edad de abandono, años de uso del tabaco, número de cigarrillos al día y dependencia nicotínica. Así mismo, se evaluó la presencia de repertorios comportamentales relacionados con la regulación personal, tales como el autocontrol, entendido como habilidad para controlar la interferencia derivada de eventos internos, medido a través del Cuestionario de Autocontrol de Rosembaum, (Capafóns, 1989) y la inflexibilidad psicológica, o incapacidad para estar en contacto con eventos privados funcionalmente aversivos, ya sean sensaciones, emociones, recuerdos, pensamientos etc. y la ejecución de conductas que alteren la forma y/o frecuencia de dichos eventos, medida con el Cuestionario de Aceptación y Acción (AAQ-II); versión española de Ruiz, Langer, Luciano, Cangas y Beltrán (2013). A los participantes que afirmaron seguir abstinentes se les evaluó el monóxido de carbono $(\mathrm{CO})$ en aire espirado mediante un cooxímetro marca Bendfont, debiendo arrojar un resultado igual o inferior a $5 \mathrm{ppm}$ (partículas por millón) para ser asignados al grupo de exfumadores.

Se usaron los estadísticos Z de Kolmogorov-Smirnof y $t$ de Student para contraste de medias aritméticas.

Se seleccionaron 137 participantes, de los cuales 99 $(72,2 \%)$ se mantenían abstinentes en el momento de la entrevista por un periodo superior a seis meses, exfumadores, o fumadores en activo que habían estado al menos seis meses, sin consumir tabaco mediante la estrategia de autoabandono, $38(27,7 \%)$, recidivantes.

De todas las variables relacionadas con el historial como fumador usadas para el presente estudio, solo la edad media de abandono resultó ser significativa, siendo mayor

Recibido: Diciembre 2016; Aceptado: Diciembre 2016.

Enviar correspondencia a:

Bartolomé Marín Romero. Unidad de Drogas y Adicciones. Buen Pastor nº 3. 14003 Córdoba.

Teléfono: 34 616693068. Email: bmarin@cop.es. 
Tabla 1. Variables relacionadas con el hábito tabáquico

\begin{tabular}{llll}
\hline & Exfumadores & Recidivantes & Z de Kolmogorov-Smirnof. (p) \\
\hline № cigarrillos/día: Media (DT) & $16,62(10,21)$ & $14,24(5,23)$ &, $86(, 447)$ \\
Edad de inicio en consumo de tabaco: Media (DT) & $16,45(2,80)$ & $14,88(5,50)$ & $1,33(, 056)$ \\
Años como fumador: Media (DT) & $19,29(10,91)$ & $14,99(10,77)$ & $1,28(, 76)$ \\
Edad de abandono: Media (DT) & $36,35(10,93)$ & $29,77(9,65)$ & $1,63(, 01)^{\star}$ \\
Dependencia (Fagerström): Media (DT) & $3,51(2,37)$ & $3,53(2.10)$ & $.484(, 973)$ \\
\hline
\end{tabular}

Nota. ${ }^{*} p f, 05$

en el caso de los que se mantenían abstinentes (36,35 vs. 29,77 años). Ver Tabla 1. En relación a las medidas sobre Autocontrol e Inflexibilidad psicológica, ambas mostraron ser diferentes en términos estadísticos (según el grupo de exfumadores o recidivantes $(27,72$ vs. $19,97, t=2,198 ; p £$ .001 ; y 23,46 vs. $25,36, t=-3.41 ; p £ .030$. respectivamente).

Estos resultados muestran que no hubo diferencias en gran parte de los hábitos relacionados con el consumo de tabaco, salvo en la edad de abandono, algo en lo que coincide con los resultados obtenidos en otros trabajos (Gregor y Borrelli, 2012; Raich et al., 2015) en fumadores que utilizan algún tipo de tratamiento, admitiendo como posibilidad que el aumento de la edad conlleva probablemente un aumento de las consecuencias aversivas derivadas del consumo de tabaco y ello facilitaría su abandono. En relación al autocontrol, los datos obtenidos coinciden con otras aportaciones realizadas por algunos autores acerca del beneficio que los programas de autocontrol pueden aportar al tratamiento del tabaquismo (Chiou, Wu y Chang, 2013). Respecto a la inflexibilidad psicológica podría ser un factor a tener en cuenta por su valor predictivo en el éxito en autoabandono, coincidiendo con Roales-Nieto et al. (2016). A pesar de lo anterior, hay que señalar que los participantes en el presente estudio han señalado una dependencia nicotínica baja, lo que podría facilitar el inicio del autoabandono, en línea con lo señalado por Linchestein y Cohen (1990). Los datos obtenidos pueden presentar algunas limitaciones debido al posible sesgo en la información de los participantes, por otra parte, la selección de los participantes no ha sido aleatoria debido a la dificultad de acceder a ellos. Los tratamientos que incluyan el abordaje del autocontrol y la inflexibilidad psicológica podrían aumentar las tasas de éxito en los tratamientos psicológicos de tabaquismo.

\section{Reconocimientos}

Este estudio ha sido financiado por el Ministerio de Economía y Competitividad, Plan Nacional de Investigación (Ref: PSI2011-24512) y ha sido dirigido por el segundo autor.

\section{Conflicto de Intereses}

Los autores declaran la inexistencia de conflictos de intereses.

\section{Referencias}

Bjartveit, K. y Tverdal, A. (2009a). Health consequences of sustained smoking cessation. Tobacco Control, 18, 197205. doi:10.1136/tc.2008.026898.

Capafóns, A. (1989). Competencia aprendida-II- (La aproximación de Rosenbaum). Fiabilidad y validez de su medida. Crítica y recomendaciones. Revista Española de Terapia del Comportamiento, 7, 18-39.

Chiou, W., Wu, W. y Chang, M. (2013). Think abstractly, smoke less: A brief construal-level intervention can promote self-control, leading to reduced cigarette consumption among current smokers. Addiction, 108, 985992. doi:10.1111/add.12100.

Gregor, K. y Borrelli, B. (2012). Barriers to quitting smoking among medically ill smokers. Journal of Behavioral Medicine, 35, 484-491. doi:10.1007/s10865-011-9376-y.

Lichtenstein, E. y Cohen, S. (1990). Prospective analysis of two modes of unaided smoking cessation. Health Education Research, 5, 63-72. doi:10.1093/her/5.1.63.

Raich, A., Martínez-Sánchez, J.M., Marquilles, E., Rubio, L., Fu, M. y Fernández, E. (2015). Smoking cessation after 12 months with multi-component therapy. Adicciones, 27, 37-46.

Roales-Nieto, J.G., San Pedro, E.M., García, R. C., Romero, B.M., López, F.J., Luciano, A. G., ...López, M.H. (2016). Smoking self-quitting and psychological flexibility. International Journal of Psychology and Psychological Therapy, 25, 123-129.

Ruiz, F. J., Herrera, 1. I. L., Luciano, C., Cangas, A. J. y Beltrn, I. (2013). Measuring experiential avoidance and psychological inflexibility: The spanish version of the acceptance and action questionnaire-II. Psicothema, 25, 123-129.

Schachter, S. (1990). 'Debunking myths about self-quitting: Evidence from 10 prospective studies of persons 
who attempt to quit smoking by themselves': Reply. American Psychologist, 45, 1389-1390. doi:10.1037/0003066X.45.12.1389.

Smith, A. L., Chapman, S. y Dunlop, S. M. (2015). What do we know about unassisted smoking cessation in Australia? A systematic review, 2005-2012. Tobacco Control, 24, 18-27. doi:10.1136/tobaccocontrol-2013-051019.

Thomsen, T., Villebro, N. y Mller, A. M. (2014). Interventions for preoperative smoking cessation. The Cochrane Database of Systematic Reviews, (3), CD002294. doi:10.1002/14651858.CD002294.pub4. 